\title{
L'aménagement du Saint-Laurent et le projet de Lachine
}

\section{The Saint Lawrence development and the Lachine project}

\author{
PAR H. CAS'TELNAU
}

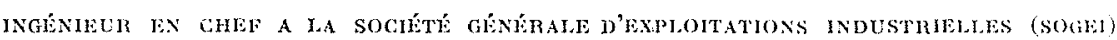

\begin{abstract}
Le bassin da Saint-Laurent, émissare des Grands Lacs, se trouve an centre d'une région fortement industrialisée de l'Amérique du Nord. Le cours du Saint-Laurent est jalonné de rapides; les travani: de canalisation ont commencé très tot et se sont développés par étapes successives. L'étape achalle, qui fait suite da de longues négociations entre less pays riverains, Etats-Cnis et Canada, créerd me véritable «Voie Maritime" de $8,20 \mathrm{~m}$ de tirant d'ean. l.es echuses, de $25 \mathrm{~m}$ de large et $820 \mathrm{~m}$ de long, permeltront ana: nabires océaniques de remonter jusqu'aux ports des frrands Lacs.

Parallelement se poursuit l'exploitation des ressontes hydrodeclriques, asines du Niagara, usine de Barnhart, usine de Beauharnois, dont les puissances dépassent $1000000 \mathrm{~kW}$.

Le projet d'aménagement de Lachine compléterail l'equipement du Saint-Laurent en utilisant all noisinage mine de Montrial ane chate de 10 metres ato mon paissance de l'ordre de $700000 \mathrm{~kW}$.

D'imporlanis trauaux de reconnaissance ont été exéculés. Cet aménagement pose notamment un problime de conpure de la rividre et surtout un probleme poir le fonctionnement en hiver. Le Saint-Laurent gele pendant 3 mois ef il faut biviter mane surelenation du plan d'eau amont an moment de la formafion de la conche de glace.
\end{abstract}

The Saint-Labrence river basin, emmisary of the Great Lakes, is situated in the center of a highly indastrialized region of North America. the Saint-Latrence river contains many rapids; the canalization of the river was started pery early and developed by successive phases. The present phase, the result of long negotiations between the United states and Canada, will open a neritable "Seaway" habing a depth of 8.20 meters. The lokk, 25 melers wide and 820 meters long, will allow ocean going vessels to navigate $n p$ to the ports of the Great Lakes. rarallel to the above construction, the development of hydroelectris resources is being carried on Niagara, Barnhart and Beauharnois plants, each excesding a capacily of $1,000,000 \mathrm{~kW}$.

The Lachine hylroelectric development will complete the Saint-Lawrence scheme by utitizing a water head of 10 meters, in the neighbourhood of Montreal, with a capacily of approximalely $700,000 \mathrm{~kW}$.

Extensive investigalions have been carried oul. This development presents namely a problen of river diversion and above all operation during the winter priod. The Saint-Lamence freezes over during 3 months and it is necessary to avoid a rise in the water level upstream during the formation of the layer of ice.

\section{L'AMENAGEMENT DU SAINT-LAURENT}

L'ensemble hydrographique des Grands Lacs et du Saint-Laurent se situe au centre d'une région industrielle très développée de l'Amérique. du Nord.

Du cólé Canada, elle comprend l'essentiel des deux provinces les plus peuplées et les plus riches : le Québec et l'Ontario, avec les grandes métropoles industrielles de Montríal el Toronlo. Du côté Etats-Unis, les Etals riverains sont New-York, Pennsylvanie, Ohio, Michigan, Illinois, avec notamment les grandes villes de Rochester, Buffalo, Cleveland, Detroit et Chirago.

Les transports par eau ont jouc un rôle es- 


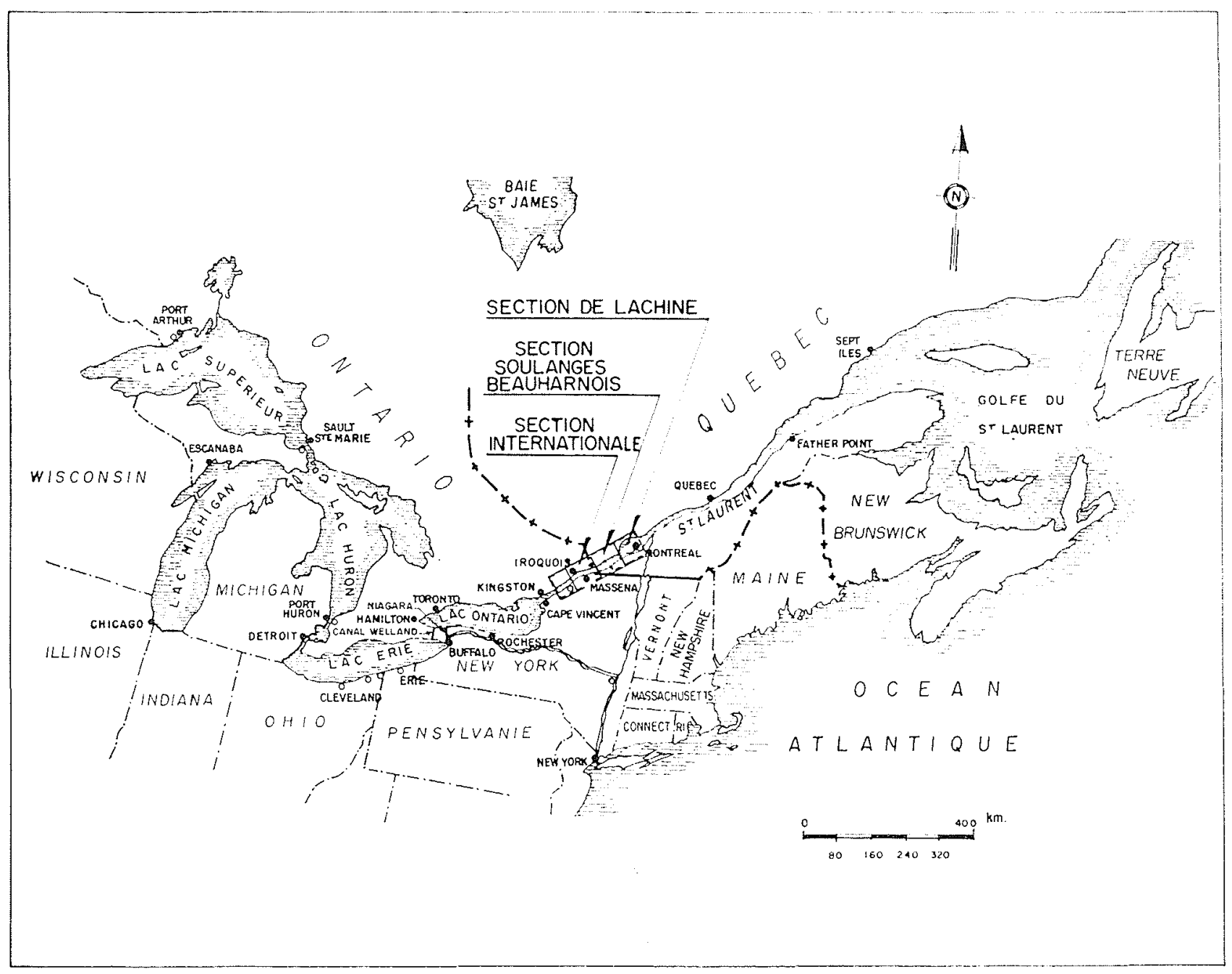

Fic. 1. - Carte du bassin du Saint-laurent.

sentiel dans le développement de cette région et en particulier l'aménagement du sillon fudson-Mowalk a été l'origine de l'énorme prospérité du port de New-York.

L'aménagement actuel du Saint-Laurent est destiné à donner une nouvelle impulsion au développement économique de cette région et notamment à la zone canadienne.

Les travaux en cours ont pour but l'établissement d'une Voie Maritime en eau profonde et l'exploitation des ressources hydroélectriques encore disponibles.

\section{Le bassin du Saint-Laurent.}

Le bassin versant des Grands Lacs est approximativement de $780000 \mathrm{~km}^{2}$ dont un tiers est occupé par la surface des lacs, qui jonent donc un rôle régularisateur considérable.
$40 \%$ des apports, comprenant les apports des rives augmentés des pluies sur les lacs, sont perdus par évaporation.

Les apports varient dans des proportions considérables; les statistiques montrent qu'il y a eu des apports nets excédant $22000 \mathrm{~m}^{3} / \mathrm{s}$, alors que pour certains mois secs l'évaporation a dépassé les apports.

Les variations saisonnières sont également importantes : les moyennes d'avril et mai sont supérieures à $14000 \mathrm{~m}^{3} / \mathrm{s}$, alors que la moyenne de novembre est de $500 \mathrm{~m}^{3} / \mathrm{s}$.

Malgré ces énormes différences, les débits écoulés se sont maintenus depuis près d'un siècle entre les limites de 5000 à $9000 \mathrm{~m}^{3} / \mathrm{s}$.

Le débit moyen à la sortie du lac Ontario est de $7000 \mathrm{~m}^{3} / \mathrm{s}$.

Les Grands Lacs constituent la source du Saint-Laurent et forment avec lui une voie d'eau s'étendant de l'intérieur du continent à la mer; 




Fili, 2.

vie perspective (ie la Voie Maritime. cntre les ports situés au fond des lacs et l'embouchure du Saint-Laurent, la distance totale est de $3500 \mathrm{~km}$.

Le Lac Supérieur se décharge dans le lac $\mathrm{Hu}$ ron par les rapides de Sault Sainte-Marie avec une différence de niveau de $6,40 \mathrm{~m}$.

Le lac Huron et le lac Michigan communiquent largement et sont sensiblement au même niveau; le lac Huron se déverse dans le lac Erié par la rivière Saint-Clair avec une différence de niveau de $2,50 \mathrm{~m}$.

Le lac Erié est relié au lac Ontario par la rivière Niagara, bien connue par ses chutes. La différence de niveau entre les deux laes est de $100 \mathrm{~m}$, dont 50 sont concentrés dans les chutes propremient dites.

La navigation entre le lac Erié et le lac Ontario emprunte depuis longtemps un canal artificiel, parallèle à la rivière Niagara et situé à l'ouest de celle-ci. C'est le canal Welland, situé entièrement en territoire canadien et qui a été progressivement approfondi.

Du lac Ontario à Montréal, il y a $300 \mathrm{~km}$, avec une différence de niveau de $70 \mathrm{~m}$, et $250 \mathrm{~km}$ de Montréal à Québec avec une différence de niveau de $6 \mathrm{~m}$. 
C'est le parcours situé entre le lac Ontario el Montréal qui fait essentiellement l'objet actuellement des aménagements constituant la nouvelle Voie Maritime.

Il convient de distinguer, de l'amont à l'aval : -..- I a Section Internationale, sur $180 \mathrm{~km}$ environ, où le Saint-Laurent fait frontière entre le Canada, province de l'Ontario, et les Etats-Unis, état de New-York.

Sur les 70 derniers kilomètres, se trouvent les rapides internationaux entre Iroquois et Barnhart-Cornwall avec principalement les rapides de Long Sault; la différence de niveau entre les extrémités de ce troncon est de l'ordre de $27 \mathrm{~m}$.

Plusieurs canaux situés en territoire canadien contournent actuellement les différents rapides de cette section.

-... La Section de Soulanges, de $26 \mathrm{~km}$ de long, qui fait suite au lac Saint-François
$(47 \mathrm{~km})$, où le Saint-Laurent pénètre dans la province de Québec.

Les rapides de Soulanges, situes entre le lac Saint-Francois et le lac Saint-Louis, sont contournés au nord par le eanal de Soulanges et au sud par le canal de Beauharnois. La différence de niveau entre les deux lacs, exploitée par la centrale de Beauharnois, est de $25 \mathrm{~m}$ environ.

- La Section de Lachine s'étend sur $50 \mathrm{~km}$. Elle comprend le lac Saint-Louis qui recoit par le lac des Deux-Montagnes la plus grande partie des eaux de la rivière Ottawa, les rapides de Lachine, l'élargissement formant le bassin de Laprairie et la partie du cours du Saint-Laurent qui borde à l'est la ville de Montréal.

Les rapides sont actuellement contournés par le canal de Lachine qui relie le lac Saint-Louis au port de Montréal.

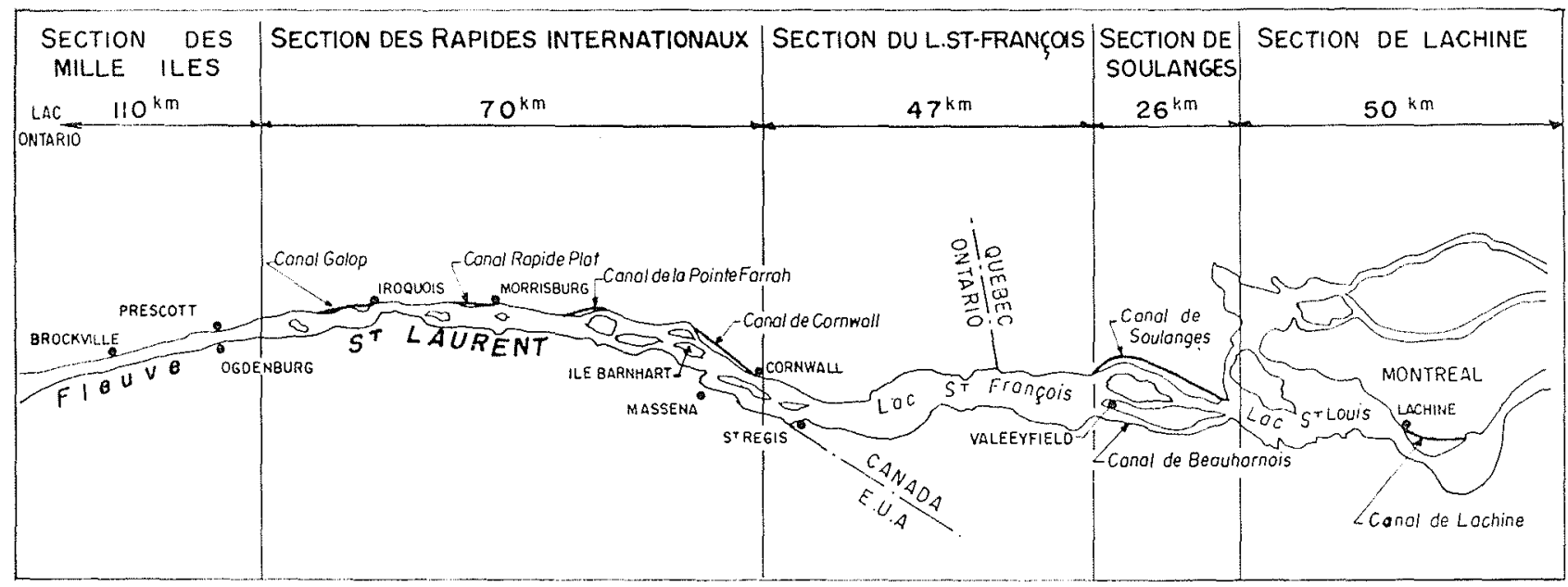

Fifi. 3. -.... Carte des différentes seclions de la Voie Maritime.

\section{Notes historiques.}

Les premiers travaux de canalisation remontent à 1700, époque à laquelle un canal de $0,50 \mathrm{~m}$ fut construit près de Lachine.

Aux environs de 1800 , il était possible de remonter au lac Saint-Louis et au lac SaintFrançois par des canaux de $0,75 \mathrm{~m}$ avec écluses de $1,80 \mathrm{~m}$ de large.

L'étape suivante, achevée en 1868, comporta la réalisation d'une voie navigable de $2,75 \mathrm{~m}$ de profondeur, comprenant notamment le canal de Lachine dans son tracé actuel, un premier canal de Beauharnois construit sur la rive sud, les canaux de la Section Internationale et le canal
Welland, ce qui permit aux navires de $42 \mathrm{~m}$ de long, $7,9 \mathrm{~m}$ de large et $2,75 \mathrm{~m}$ de tirant d'eau, de remonter pour la première fois de Montréal au lac Erié.

Un nouveau pas fut franchi aux environs de 1900 , lorsque la profondeur de la voie navigable fut portée à $4,27 \mathrm{~m}$ (14 pieds). Les travaux consistèrent en l'approfondissement d'une partie des canaux existants, le remplacement du canal de Beauharnois par le canal de Soulanges, sur la rive nord, équipé de 5 écluses, et la construction d'un nouveau canal à Welland, équipé de 26 écluses.

L'idée d'établir une voie en eau profonde allant des Grands Lacs à la mer remonte à 1905; les 


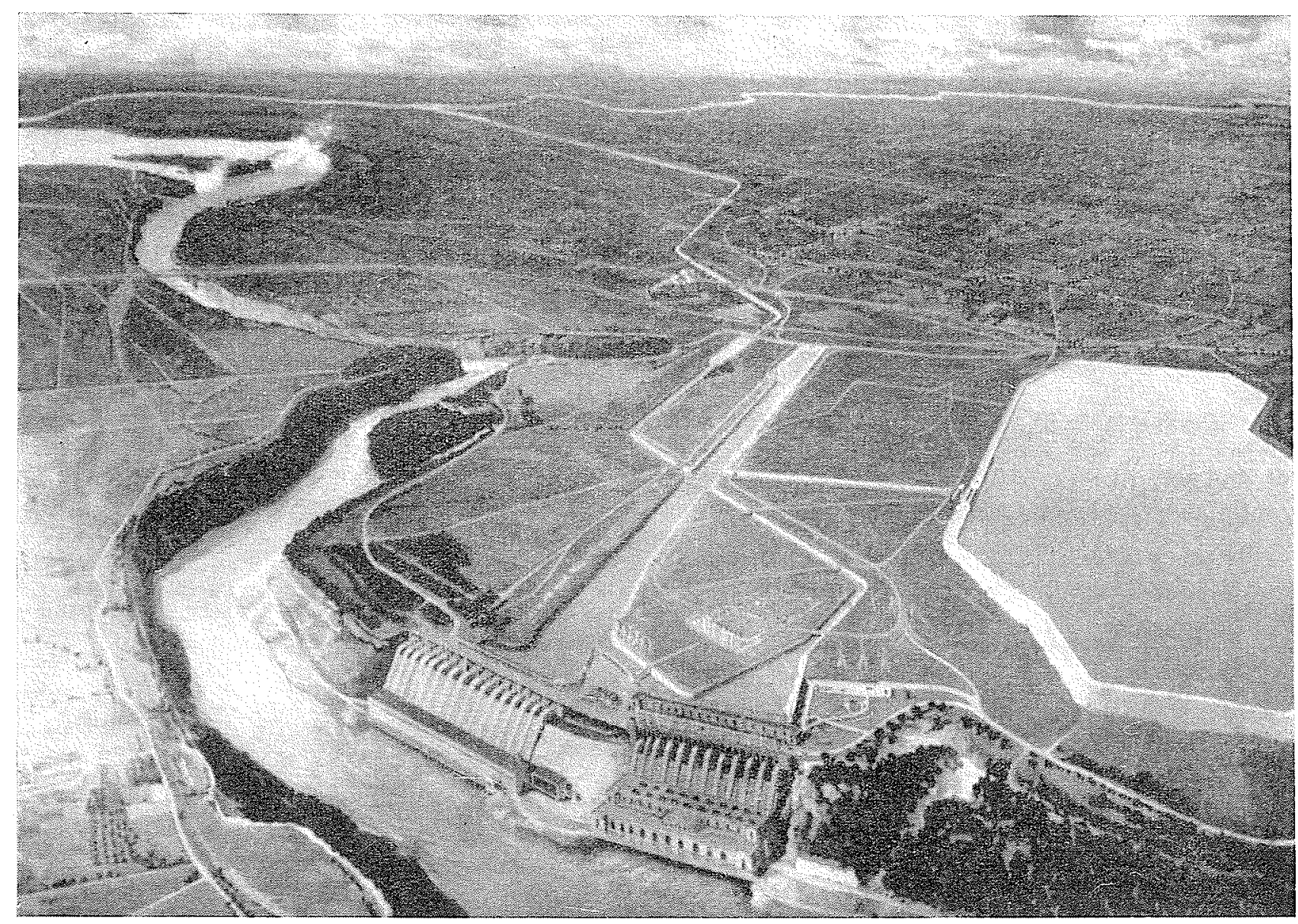

firi. 4. - Installation de Sir Adam Beck

négociations à ce sujet, entre le Canada et les Etats-Unis, assez laborieuses, furent poursuivies au coul's des années.

Le traité du 11 janvier 1909 entre les gouvernements des Etats-Unis et du Canada créa une Commission Mixte Internationale pour l'amélioration du Saint-Laurent entre le lac Ontario et Montréal, afin de le rendre navigable aux navires a fort tirant d'eau et d'assurer la meilleure utilisation de l'eau pour la production d'énergie.

Un premier rapport, précisant notamment le coût envisagé pour l'établissement d'une voie maritime de $7,62 \mathrm{~m}$ de profondeur, fut lemis a la Commission en 1921. Il était établi conjointement par deux ingénieurs, avec cependant une divergence concernant la régularisation du lac Ontario; l'ingénieur canadien recommandait une plus forte restriction des débits en hiver pour avoir de meilleures conditions an moment de la formation des glaces.

Après discussion de ce rapport, les deux pays décidèrent de constituer en 1924 une Commission mixte d'ingénieurs pour procéder à une élude complète concernant l'amélioration des condilions de navigation sur le Saint-Laurent et les problèmes annexes tels que la production d'énergie et la régularisation du lac Ontario.

Le résultat des travaux considérables entrepris par cette Commission fit l'objet d'un l'apport daté de novembre 1926 dans lequel sont précisés :

- les travaux de reconnaissance effectués (topographie et sondages);

- les études concernant la régularisation des Grands Lacs;

- les plans détaillés et estimations des projets proposés;

-.-- les études concernant les niveaux el débits à Montréal et à l'aval;

- Ia formation des glaces sur le Saint-Laurent;

- Les études expérimentales sur la résistance de la glace;

-. un programme d'exécution.

Pendant ce temps, les travaux de construction 
du canal de Welland, qui avaient été entrepris en 1913 et interrompus pendant la première suerre mondiale, furent repris et acherés en 1932. Le nouveau canal était équipé de 8 éciuses de $260 \mathrm{~m}$ de long, $24 \mathrm{~m}$ de large et $9,15 \mathrm{~m}$ de profondeur. La profondeur minimum du canal est actuellement de $7,60 \mathrm{~m}$, mais sera portée par des dragages au tirant d'eau normal de la nouvelle Voie Maritime de $8,20 \mathrm{~m}$ (27 pieds).

C'est au cours de la même année 1932 que les recommandations de la Commission mixte de 1926 furent incorporées dans le Traité de la Voie Maritime profonde du Saint-Laurent; mais ce traite ne fut pas ratifié par le Sénat américain.

De nouveaux efforts furent entrepris en 1941 pour faire approuver le projet (avec je St. Lawrence Bassin Agreement), mais sans succes.

Jusqu'à cette époque, l'étude de la canalisation avait été liće aux projets d'équipement hydroćlectrique de la Section Internationale.

Une nouvelle approche fut tentée par le Canada; le problème de la production d'énergie serait étudié séparément par des établissements compétents canadiens (Ontario Hydro) et américains; le Canada, pendant ce temps, étudierait la construction d'une voie maritime entierement anadienne.

L'exploitation de l'énergie par Ontario Hydro et l'Etat de New-York fut acceptée par la Commission Internationale en 1952.

Les choses allèrent ensuite rapidement. En juin 1953, la Commission Fédérale américaine de l'Energie octroya un permis de cinquante ans à la
Power Authority de l'Etat de New-York pour la réalisation de la moitié du projet. La décision fut confirmée en juin 1954 par la Cour Suprême des Etats-Unis.

En mai 1954, le président Eisenhower signa le Wiley Dondero Bill autorisant un organisme anćlicain à construire des écluses pour contourner les barrages près de Cornwall.

Au Canada, l'Acle de l'Autorité de la Voie Maritime exécutoire le $1^{\mathrm{rr}}$ juillet 1954 domnait pouroir à l'Autorité de procéder à la construction de la Voie Maritime, soit entierement au Canada, soit en conjonction avec les travaux entrepris par l'Autorité compétente dans les Etats-Unis.

Les travaux commencèrent aussitôt; la plus grande partie, représentant les deux tiers des dépenses, est faite au Canada et comprend cing des sept écluses prévues entre le lac Ontario et Montréal. Quatre de ces écluses sont situées dans la province de Québec.

\section{Régularisation du lac Ontario.}

Dans l'ensemble des Grands Lacs, seule la régularisation du lac Óntario a été envisagée.

Ce problème a fait l'objet de longues études par la Commission Internationale et plusieurs méthodes ont été successivement mises sur pied.

Celle qui a été retenue en 1956 portait l'indicatif 12.A.9 (M). Elle sera pent-être encoro sus-

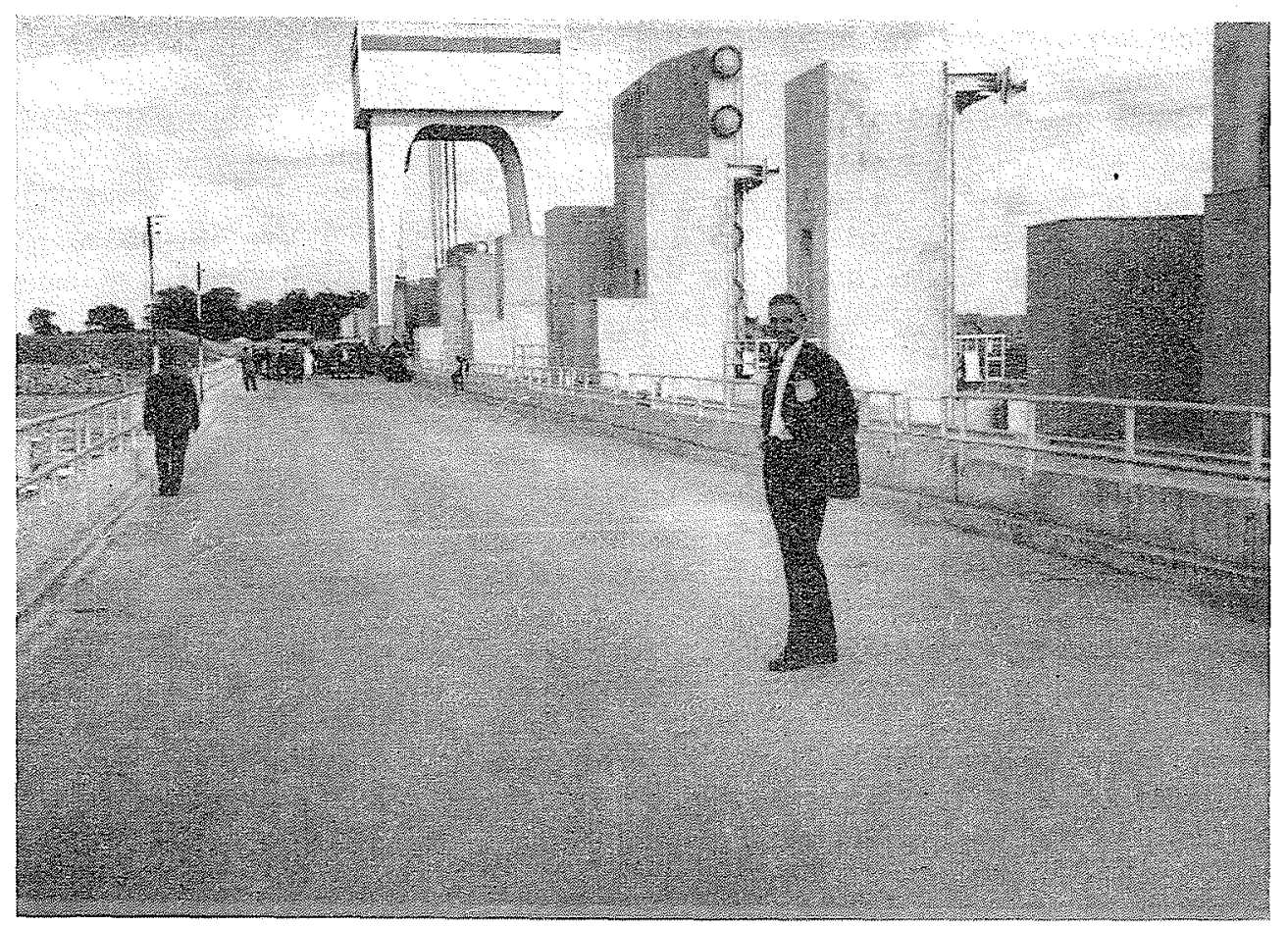




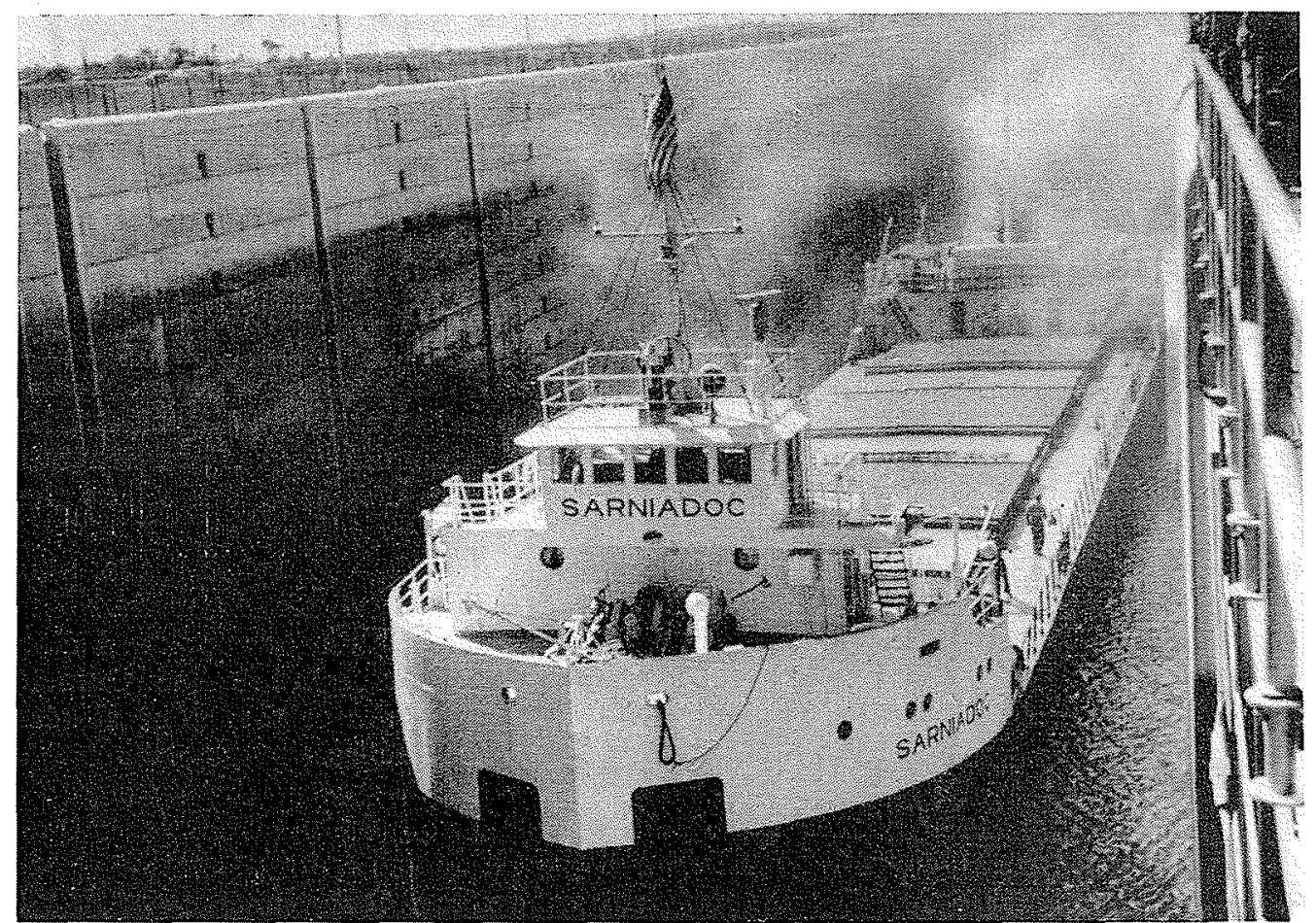

Firg. 6 .

Ecluse Eisenhower

ceptible d'ajustements de détals, mais les principes généraux subsisteront certainement.

Ces principes concernent notamment:

-..- Le maintien du niveau dans le port de Montréal aux cotes antérieures, comple tenu des dérivations d'eau faites à Chicago;

- Un écoulement, entre le 15 décembre et le 31 mars, aussi grand que possible et soutenu, de manière à minimiser les difficultés d'exploitation des aménagements hydroélectriques en période de gel;

-. Une régularisation conduite de manière à limiter les variations de niveau du lac Ontario lui-même à une amplitude de $1 \mathrm{~m}$ environ et à limiter les débits maximums et les débits qui se produiront pendant les crues de la rivière Ottawa.

L'application de ces principes se traduit par des courbes qui donnent le débit à lâcher en fonction de la cote au lac Ontario pendant les différents mois de l'année. Le problème qui a demandé le plus d'attention est celui qui concerne les lâchures au mois de décembre, époque de la formation des glaces, où il y a intérêt à limiter les débits pour éviter la formation d'embâcles, mais où les desiderata des différents exploitants ne coïncident pas, étant donné que la prise en glaces ne se produit pas en même temps dans la région de Montréal et dans la région de Cornwall.
L'étude faite donne, mois par mois, la fréquence des débits qui auraient été obtenus à la sortie du lac Ontario d'une part et à la sortie du lac Saint-Louis d'autre part, si l'on avait appliqué la méthode 12.A.9(M) avec les apports des années 1860 à 1954 .

Les débits sortant du lac Saint-Louis varient de 5500 à $13000 \mathrm{~m}^{3} / \mathrm{s}$, mais pendant la période comprise entre le 15 décembre et le 31 janvier, ils n'excederont jamais $7800 \mathrm{~m} / \mathrm{s}$. L'intérêt de cette limitation pour le projet de Lachine tient à ce que la prise en glace du Saint-Laurent se produit pendant cette période et que les risques de difficultés et d'embâcles sont ainsi linités.

\section{Caractéristiques des aménagements.}

\section{Grands Lacs :}

Le trafic très intense sur les Grands Lacs est assuré grâce aux écluses qui permettent le passage de navires du lac Erié au lac Supérieur avec un tirant d'eau de $7,60 \mathrm{~m}$ à la descente et de 6,40 à la remontée.

\section{Chutes du Niagara:}

Entre le lac Erié et le lac Ontario, le canal Welland verra sa profondeur portée de 7,62 à $8,20 \mathrm{~m}$ et sera élargi par endroits.

Le site des chutes du Niagara est exploité par les usines de Sir Adam Beck sur Ie versant ca- 
nadien. Le schéma qui résulte de la superposition de deux aménagements et d'une installation de pompage est assez compliqué (voir fig. 4).

L'installation de pompage de $280000 \mathrm{ch}$ est justifiée par les sujétions imposées pour maintenir aux chutes leur caractère touristique. Le traité impose en effet de laisser couler pendant les heures de jour de la saison touristique un débit minimum de $2800 \mathrm{~m}^{3} / \mathrm{s}$, mais permet de le réduire la nuit et aux autres époques à $1400 \mathrm{~m}^{3} / \mathrm{s}$.

La station de pompage, du type " outdoor *, est installée en bordure du bassin d'accumulation et comprend 6 groupes Francis à pales mobiles réversibles pouvant fonctionner en turbines ou en pompes. Les débits des pompes varient de 110 a $140 \mathrm{~m}^{3} / \mathrm{s}$ sous des hauteurs allant de 26 à $18 \mathrm{~m}$.

L'usine proprement dite de Sir Adam Beck II est située en bordure de la riviere Niagara; elle fournira, une fois complétée, une puissance totale de $1288 \mathrm{MW}$ sous une chute de $90 \mathrm{~m}$ en 16 groupes; l'installation est du type «indoor » et occupe une longueur de $350 \mathrm{~m}$.

Sur le versant américain, une ancienne usine de $300 \mathrm{MW}$ a été mise hors service en 1956 à la suite d'un éboulement. Une nouvelle usine, la centrale de Lewiston, est en cours de construction suivant un schéma similaire à celui de Sir Adam Beck. La station de pompage comprendra 12 groupes de $20 \mathrm{MVA}$, et la centrale, 13 groupes de $150 \mathrm{MW}$; les deux installations seront du type «outdoor».

\section{Voie maritime proprement dite.}

Elle assurera partout un tirant d'eau de $8,20 \mathrm{~m}$ (27 pieds).
Les autres caractéristiques principales sont les suivantes :

- écluses : largeur $25 \mathrm{~m}$ - longueur $280 \mathrm{~m}$;

- canaux : largeur de 60 à $120 \mathrm{~m}$ suivant les rives;

- rayon de courbure mininum : $1500 \mathrm{~m}$;

-. tirant d'air : $37 \mathrm{~m}$.

Dans les chenaux en rivière, la vitesse ne dépassera pas $1,20 \mathrm{~m} / \mathrm{s}$ et sera même réduite dans les points particuliers.

D'importantes études sur modele réduit ont été laites aux Etats-Unis et au Canada en vue de réaliser économiquement les meilleures conditions de navigation dans les différentes seclions du Saint-Laurent. Des essais systématiques ont été faits en outre pour réaliser de bonnes conditions de remplissage et de vidange des écluses et pour vérifier le fonctionnement des portessecteurs qui doivent pouvoir être fermées dans toutes les conditions en cas d'incidents survenant à l'aval.

Les principaux ouvrages réalisés pour la Voie Maritime sont les suivants, de l'amont à l'aval :

- Dragages dans la zone d'iles qui suit la sortie du lac Ontario;

- Barrage et écluse d'Iroquois : il s'agit d'un barrage-poids comportant 32 passes de $15,25 \mathrm{~m}$ de largeur, équipées de vannes-wagon et de batardeaux. Ce barrage sera utilisé pour effectuer la régularisation du lac Ontario.

L'écluse d'Iroquois a été la première de la nouvelle Voie Maritime à être mise en service.

- Le barrage de Long Sault est un barragepoids incurvé vers l'amont, de $44 \mathrm{~m}$ de hauteur,

Fig. 7.

Coupure

des rapides

de Long Sault.

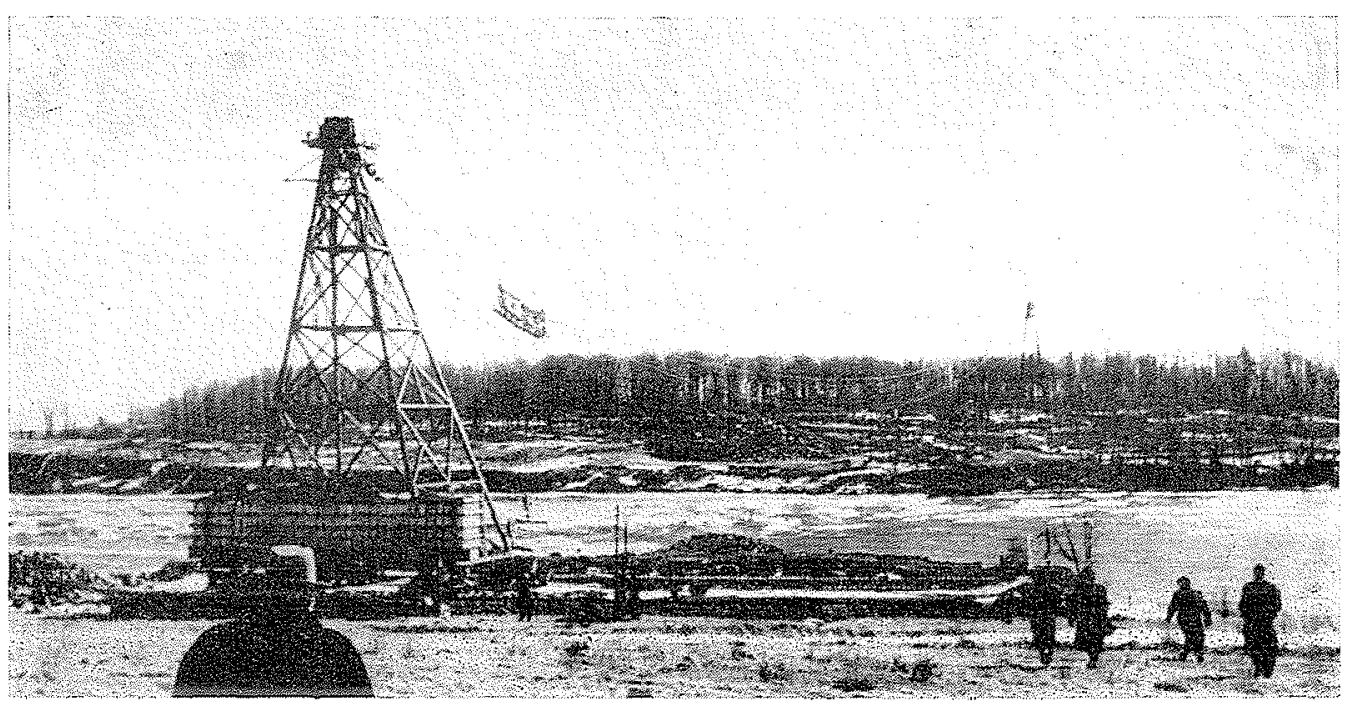




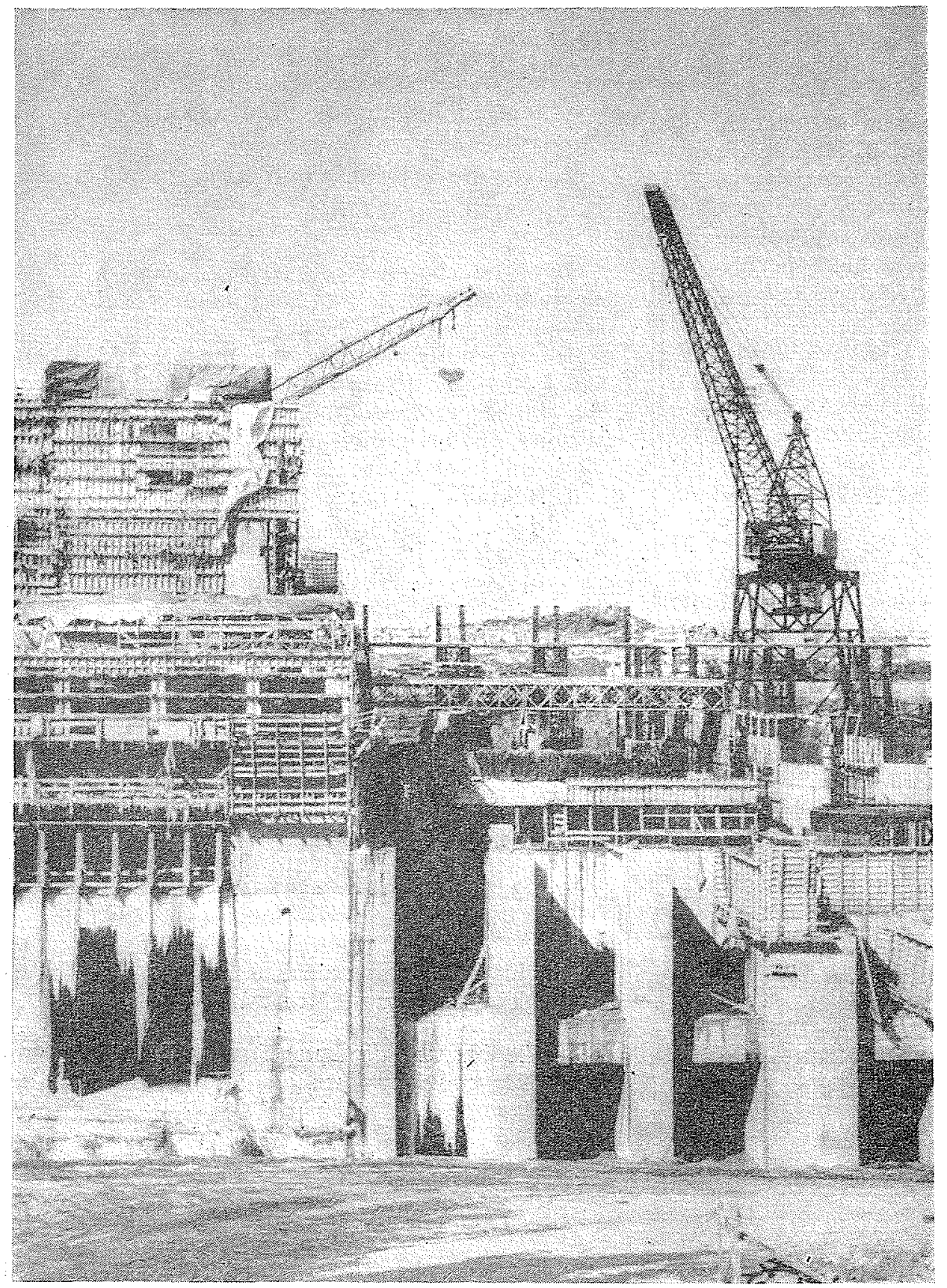

Firis. 8.

Usine de Barnlart

en cours

de construction,

en hiver.

qui comporte 30 passes déversantes de $15,25 \mathrm{~m}$ de largeur. Pour sa construction, il a été nécessaire d'effectuer une coupure dans les rapides de Long Sault à l'aide de bloes de rochers de plusieurs tonnes, mis en place par blondin.

- Canal Américain. - La navigation contourne le barrage de Long Sault par un canal de $16 \mathrm{~km}$ situs sur le territoire des Etats-Unis. Deux écluses, Eisenhower et Grass River (Snell), rattrapent une dénivellation de $27 \mathrm{~m}$.

Un nouveau pont route suspendu a été cons- truit en aval de la deuxième écluse pour remplacer une traversée mixte route et voie ferrée qui a été éliminée.

- Canal de Beauharnois. -- Dans cette zone, le Saint-Laurent est entièrement canadien. La Voie Maritime emprunte le canal usinier de $24 \mathrm{~km}$ de long, qui a été approfondi dans ce but à $8,20 \mathrm{~m}$ sur une largeur de $180 \mathrm{~m}$.

Deux écluses se partagent la chute maximum de $28 \mathrm{~m}$ qui peut exister entre le lac Saint-Francois et le lac Saint-Louis. 


\section{- Zone de Lachine.}

Le lac Saint-Louis est traversé en eaux profondes, et le canal proprement dit reprend sur la rive sud du Saint-Laurent dans la partie aval du lac.

Un prenier troncon de $13 \mathrm{~km}$ conduit à l'écluse de la Côte Sainte-Catherine, où la dénivellation moyenne est de $10 \mathrm{~m}$.

Un second troncon de 13 kim aboutit à l'écluse Saint-Lambert où la dénivelée est de 4 m.

L'extrémité aval du canal débouche dans to Saint-Laurent en face du port de Montréal.

Les ponts routier's Honoré-Mercier el facques-Cintier sont relevés. Les ponts mixtes et de chemin de fer ont été équipés de travées mobiles.

\section{Equipements hydroélectriques et travaux annexes.}

- Usine de Barnhart. -- Cet aménagement, partagé entre l'Etat de New-York et la Province de l'ontario, exploite la dénivelée d'environ
27 créée par la retenue de Long Sault. Il comprend deux fois 16 groupes hélice donnant une puissance totale de $1640 \mathrm{MW}$. L'usine est du type «outdoor » avec convercles hermétique; en deux parties, mais eomporte deux travées de montage couvertes aux extrémités, où pénètrent les portiques de manœurre.

Aux extrémités et au centre de l'usine ont été prevus des pertuis pour l'évacuation des glaces.

Les travaux ont nécessité la construction d'un batardeau cellulaire en palplanches, de plus d'un $\mathrm{km}$ de long. Les bétomnages ont éte pratiquement interrompus en hiver du côté américain, alors qu'ils étaient poursuivis presqu'à la même cadence du côté canadien.

-- Usine de Beauharnois. - Cette usine, commencée il y a de nombreuses années, exploite la différence de niveau créée par les rapides de Soulanges entre le lac Saint-Francois et le lac Saint-Louis. Elle a été construite en trois étapes; les deux premieres comportaient 26 groupes Francis représentant environ $1000 \mathrm{MW}$. Une extension est en cours avec 11 groupes hélice

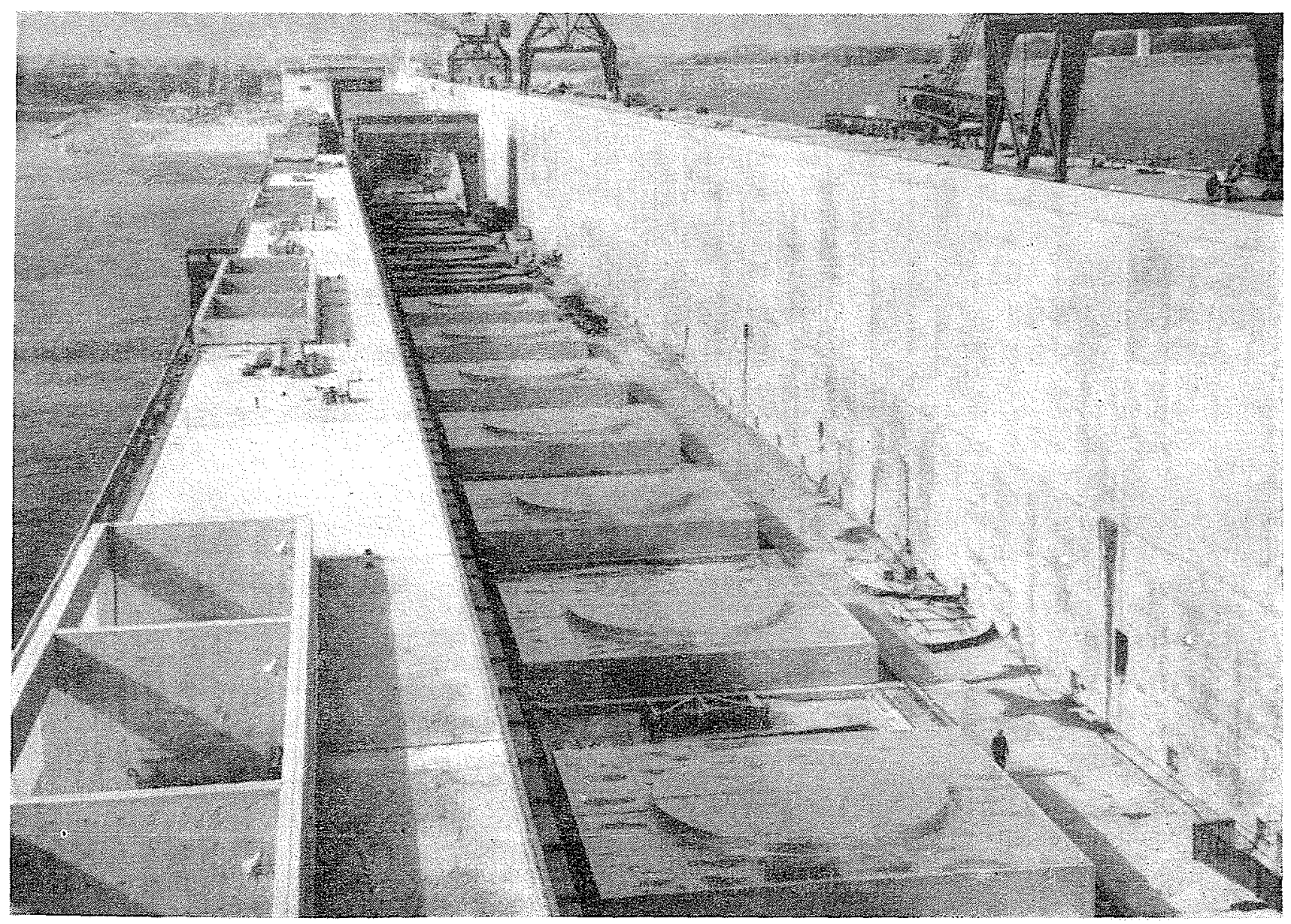

Fig, 9. -..- Lsing do Banhat en voie d'achevement, 


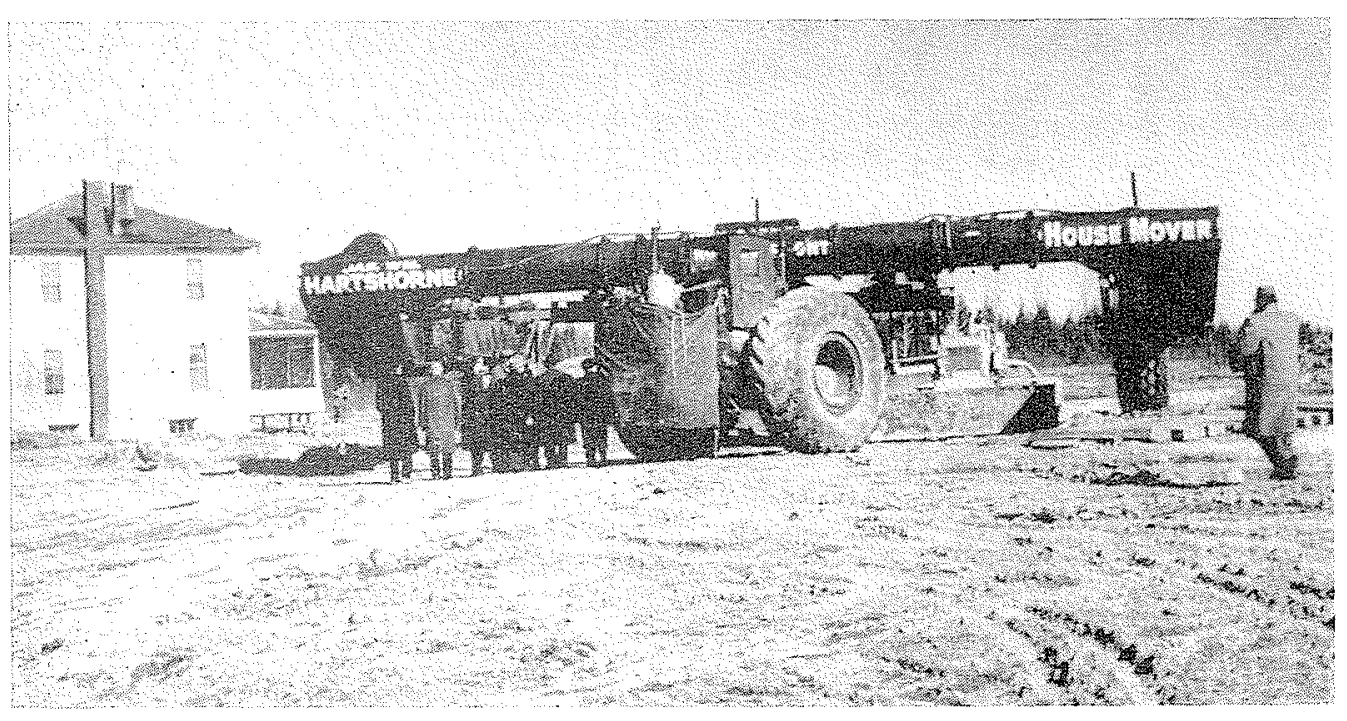

Fig. 10 .

Machine à déplacer

les maisons

« House Mover

donnant une puissance supplémentaire d'environ $500 \mathrm{MW}$. L'usine est du type « indoor »; le bâtiment achevé mesurera plus d'un km de long.

- Travanx annexes. - La construction de la Voie Maritime et la création de la retenue de Banhart ont entraîné des travaux considérables de déplacements de routes, voies de chemins de fer, lignes électriques et modifications de ponts.

Plusieurs agglomérations ont dû être déplacées. Dans un grand nombre de cas, ce sont les maisons elles-mêmes qui ont été transportées à l'aide d'une machine spéciale, le "House mover».

\section{Exécution des travaux.}

Les quelques chiffres de prévisions suivants donneront une idée de l'importance des travaux.
Voie Maritime :

Dragages........... $14000000 \mathrm{~m}^{3}$

Excavations........ . $\quad 39000000 \mathrm{~m}^{3}$

Bétons........... $1500000 \mathrm{~m}^{2}$

Aciers............ $45000 t$

Palplanches.......... $\quad 10000 \mathrm{t}$

Main-d'œuvre....... 5500

Aménagement hydroélectrique de Banhart:

Excavations......... $70000000 \mathrm{nin}^{3}$

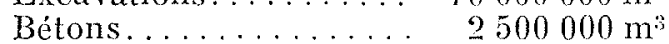

Aciers............ $28000 \mathrm{t}$

Main-d'œuvre....... 10500

Les coûts prévus étaient, pour la Voie Maritime, de $130000000 \$$ côté américain et 260000000 sôté canadien; pour l'aménagement hydroélectrique, $600000000 \$$.

Les travaux ont commencé en aout 1954 et l'inauguration officielle est prévue pour le printemps 1959.

\section{LE PROJET DE LACHINE}

MM. Cartier, Côté, Pietle, ingénieurs-conseils à Montréal, ont été chargés par la Commission Hydroélectrique de la Province de Québee ( $\mathrm{Hy}-$ dro-Québec) d'une étude préliminaire de cet aménagement, à laquelle ils ont bien voulu associer la SOGEI.

L'aménagement tire son nom des rapides de Lachine, situés au sud de l'Ile de Montréal. Ce sont ces rapides qui ont arrêté Jacques Cartier et les premiers navigateurs français remontant le cours du Saint-Laurent. A l'époque, ceux-ci croyaient pénétrer en Asie et le nom de Lachine vient de cette croyance.
Sur le cours du Saint-Laurent, la section dite de Lachine s'étend du lac Saint-Louis au port de Montréal.

La dénivelée totale est de $15 \mathrm{~m}$ et se divise en deux marches:

- La première, d'environ $10 \mathrm{~m}$, se situe entre le lac Saint-Louis et le bassin de Laprairie et correspond essentiellement aux rapides de Lachine;

- La seconde, à peine $5 \mathrm{~m}$, provient des sections étroites situées en aval du bassin de Laprairie. 
Des projets anciens avaient envisagé l'exploitation en une seule chute de la différence de niveau lotale. Cette solution aurait conduit à construire d'énormes digues le long des rives du Saint-Laurent et à avoir une partie de la ville de Montréal en contrebas de la retenue. Elle parait maintenant abandonnée.

L'aménagement actuellement étudié ne vise que l'équipement de la première marche, celle des rapides de Lachine; son intérêt serait de fournir une puissance hydroélectrique très inportanle au voisinage immédiat du grand centre industriel de Montréal.

Les principales caractéristiques sont les suivantes :

\section{HYDROLOGIE :}

Le Saint-Laurent ne recoit pas d'affluents notables entre le lac Ontario et le lac Saint-Louis, mais, à la sortic de ce dernier, il est grossi de la plus grande partie de la rivière Ottawa, dont le complément s'écoule par les rivières des Prairies ef des Mille Iles situées au nord de l'île de Montréal.

Les débîts de la rivière Ottawa, bien moins régulière que le Saint-Laurent, varient entre 800 et $7000 \mathrm{~m}^{3} / \mathrm{s}$.

Compte tenu de cet apport et de la régularisation du lac Ontario, le débit disponible à Lachine variera de 5500 à $13000 \mathrm{~m}^{3} / \mathrm{s}$; la moyenne est de l'ordre de $8000 \mathrm{~m}^{3} / \mathrm{s}$ et la crue maximuni envisagée d'environ $16000 \mathrm{~m}^{3} / \mathrm{s}$.

\section{IMPLANTATION ET TOPOGRAPHIE :}

Pour bénéficier de toute la chute disponible, l'usine électrique devra être implantée à l'aval des rapides, à l'entrée du bassin de Laprairie. A hauteur de cet emplacement, le Saint-Laurent se divise en deux bras larges de près d'un km chacun, entourant l'île au Héron.

La retenue s'étendra à l'amont jusqu'au lac Saint-Louis et réagira sur le niveau de celui-ci. Dans la portion rétrécie du cours du fleuve, elle sera bordée au sud par la digue de la Voie Maritime sur une dizaine de $\mathrm{km}$, et au nord par la rive naturelle à l'amont et par une digue à construire sur environ $5 \mathrm{~km}$ à l'aval, à partir de la prise d'eau de l'aqueduc de Montréal.

Les travaux de topographie effectués à l'occasion de cette étude ont compris l'établissement d'un réseau de triangulation, matérialisé sur le terrain et encerclant toute la zone intéressée par les travaux.

Tous les terrains bordant le Saint-Laurent, y compris l'lle au Héron et l'lle du Diable ont été levés et restitués à l'échelle du $1 / 1200$.

\section{GÉologie :}

Les terrains sous-jacents, qui datent du primaire, sont constitués par des calcaires dans la partie amont de la retenue et par des schistes siliceux dans la partie aval et la zone de l'usine; les couches sont sensiblement horizontales.

Les schistes constituent une bonne fondation,
Fig. 11 .

Construction

d'un «crib»

au Tac Saint-Louis.

Elément de digue

constitué

par une sorte

de caisse en bois,

lestée avec

des enrochements.

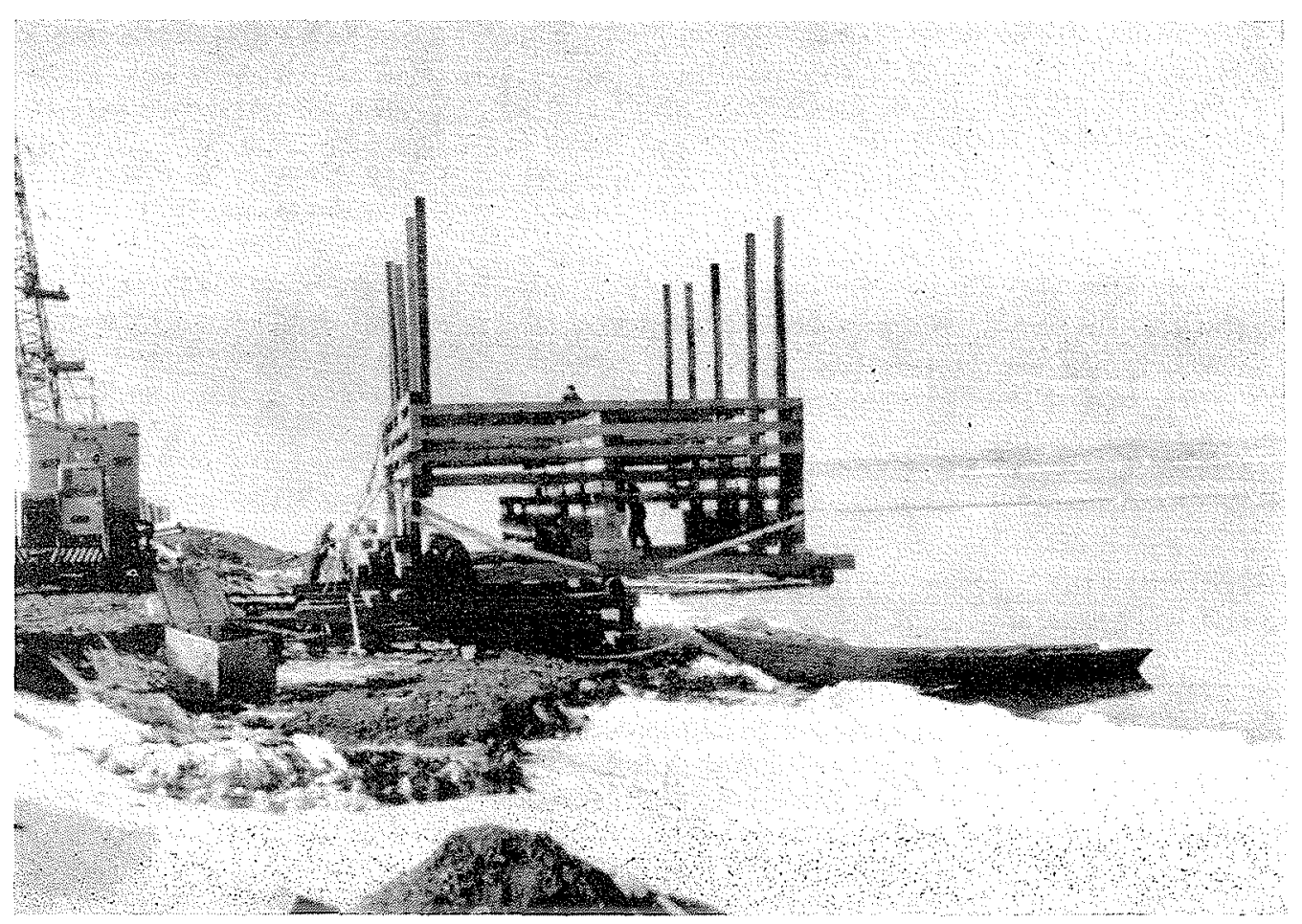




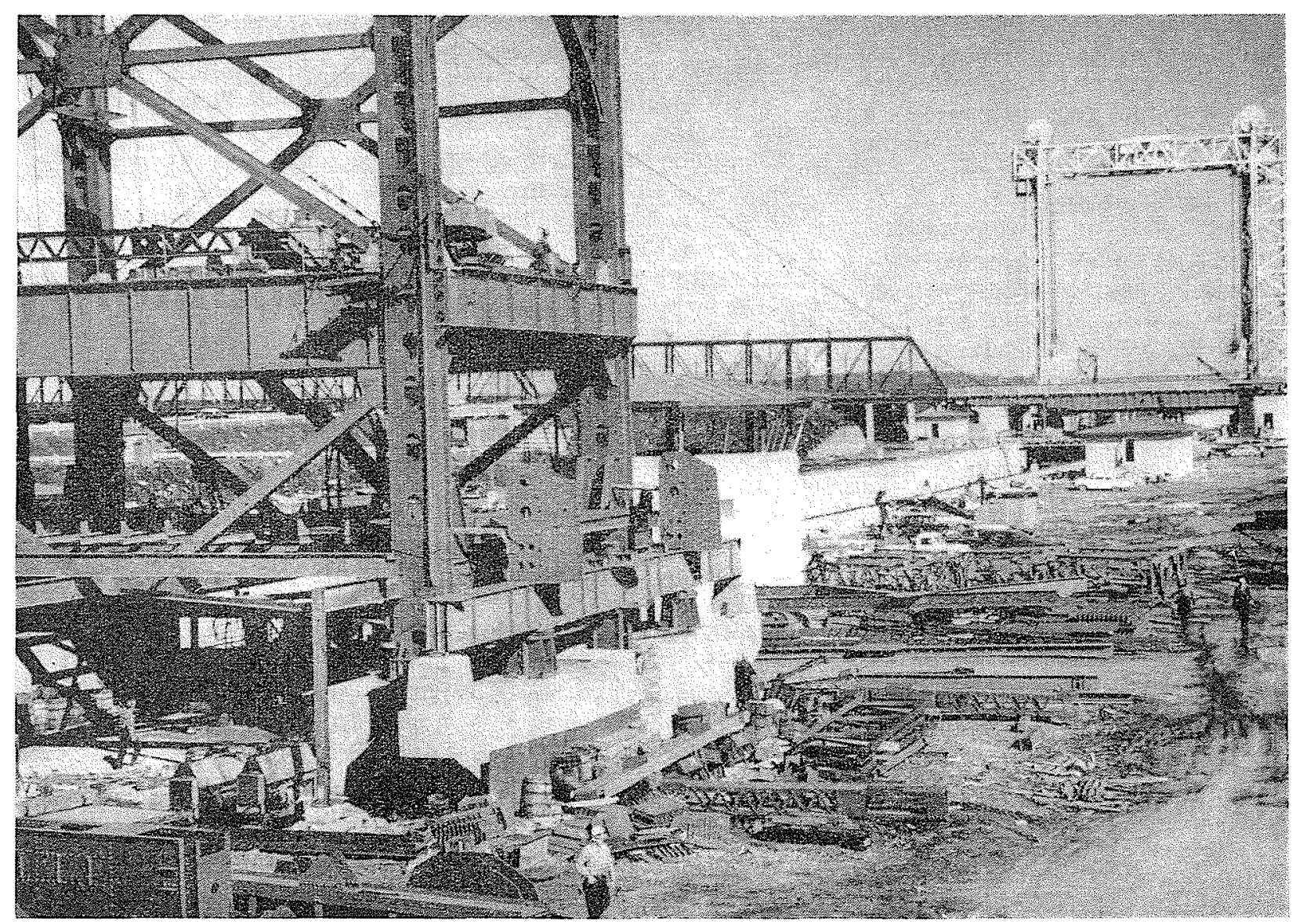

Fili. 12.

Pont levant au-dessus

de l'écluse

Saint-Lambert

mais peuvent ètre fracturés et perméables sur. les premiers metres de profondeur.

Au cours du secondaire, la mise en place de la masse intrusive qui a créé la colline de MontRoyal, a été accompagnée de l'introduction dans les schistes d'abondants filons de roches ignées.

Ces filons, qui traversent la rivière à hauteur de llle au Héron, sont beaucoup plus résistants a l'érosion fluviale que les schistes: ils ont empêché le fleuve d'atteindre son profil d'équilibre et formé les rapides de Lachine.

Les terrains de couverture que l'on rencontre sont des dépôts quaternaires et en grande. partie des moraines glaciaires qui constituent un excellent matériau de digue. On trouve également du silt et des alluvions.

Les travaux de reconnaissance ont comporte l'exécution de relevés géologiques, sondages, essais de perméabilité, puits et tranchées, et se sont étendus à la rive sud (où ils completaient les renseignements déjà obtenus pour la Voie Maritime), au bras sud dans la zone prévue pour l'implantation de l'usine, à l'Ile au Héron et à la rive nord dans la zone aval. Dans celte dernière zone, l'un des buts recherchés était d'étudier le comportement de la nappe phréaitgue.

\section{Etude du cours dans la zone des rapides.}

Les rapides de Lachine constituent le déversoir du lac Saint-Louis. La section de contrôle des débits est formée par une sorte de seuil qui traverse le fleuve vers l'extrémité amont de l'Ile au Héron et à hauteur de l'lle du Diable.

Entre ce seuil et le lac Saint-Louis, il est possible de circuler en harque et il a été relativement facile de sonder les fonds.

A l'aval, où se trouvent les rapides proprement dits, les relevés sont plus difficiles à obtenir. En fait, les rapides sont formés dans chaque bras par une bande de largeur limitée, à fort courant (4 à $5 \mathrm{~m} / \mathrm{s}$ ) avec intumescences; Je reste $d u$ cours est constitué de zones d'eau peu profondes et à courant plus lent.

Il est pratiquement impossible d'accéder à cerlaines des zones à fort courant.

- Les relevés des fonds correspondants ont pu ètre obtenus par une méthode qui avait été précédemment utilisée en amont des chutes du Niagara et aux rapides de Long Sault et qui comporte l'emploi d'un hélicoptère.

Le procédé consiste à descendre un plombsonde suspendu à un filin très fin d'environ $300 \mathrm{~m}$ de longuenr maintenu sous tension par 
son treuil. Un câble est attaché sur le filin à une distance connue du plomb-sonde.

Pour effectuer un sondage, on laisse tomber le jlomb à l'endroit voulu, le câble étant tendu verticalement par l'hélicoptère et le treuil. La position et la cote de la cible sont données par l'intersection de visées faites à partir de quatro stations installées sur les rives; on en déduit aisément les coordonnées du poinl sondé.

Il a été nécessaire d'effectuer des corlections pour tenir compte de la poussée de l'eau sur Io filin; ces corrections ont été faites en se basant sur des relevés effectués dans des zones où les fonds étaient commus.

Les équipes utilisées pour ces mesures comprenaient plus de 20 personnes.

- Les vitesses de courant ont été déterminées a l'aide de houces lancées de l'amont. Les positions de ces bouées étaient repérées à intervalles réguliers d'une minute à parlir de slations situées sur les rives.

Ces mesures ont été complétées daus les zones accessibles par des levers de profils en travers el des mesures de vitesses faites au moulinet.

-.- Les lignes d'eau déjà connues au lac SaintLouis et au bassin de Laprairie, ont été précisées par l'installation d'échelles limnimétriques sur les deux rives et de part el d'autre de l'Ile an Héron.
-_- A l'aide de ces différents renseignements, on s'esi efforcé de déterminer la répartition des débits entre les bras situés au nord et au sud de l'lle au Héron; il semble que plus de $60 \%$ du dóbit passe dans le bras sud.

L'ensemble de ces informations itait recherché en vue de l'établissement des modèles réluits ef de l'étude des coupures.

\section{Usine et barrage.}

Différentes variantes d'usines « outdoor » et " indoor » ont été examinées au cours de l'étude próliminaire.

Les indications suivantes ont simplement pour bul de donner une idée de ce que pourait ètre laménagement de celte chute:

Puissance equipée de lordre de 700 MW. Produetibilité dépassant 5 milliards de kWh.

-..- Equipement de 24 groupes Kaplan de $340 \mathrm{~m} \% \mathrm{~s}$ sous $10,5 \mathrm{~m}$ de chute, tournant à $69,3 \mathrm{tr} / \mathrm{mn}$ ct fournissant $28 \mathrm{MW}$ chacun. Une étude plus poussée ponrrait montrer l'intérèt de prévoir linstallation de deux groupes supplémentaires.

- Groupes liés deux par deux en unités électriques et branchés sur 12 transformateurs de $70 \mathrm{MVA}, 13,8 / 110 \mathrm{kV}$ (celle dernière tension est celle qui existe autour de Montréal).




- Implantation de la centrale dans le bras sud, où le bâtiment d'usine occuperait une longueur de $800 \mathrm{~m}$ environ.

- Evacuateurs dimensionnés pour la crue totale de $16000 \mathrm{~m}^{3} / \mathrm{s}$ et réalisés sous la forme de 24 passes de $15 \mathrm{~m}$ de large, largeur économique très généralement adoptée dans les équipements de la région, aved une lame d'eau maximum de $8,5 \mathrm{~m}$.

-.-- Passes réparties entre les bras nord et sud, équipées soit de vannes-wagon, soit de vannes-secteur; une partie des vannes serait a commande individuelle, les autres commandées par portique.

--.- Durée envisagée pour les travaux : 5 a 6 ans; les premiers groupes étant mis en service au cours de la quatrieme annce

\section{Problème des coupures.}

Les deux bras du Saint-Laurent situés de part et d'autre de l'Ile au Héron devront être coupés successivement et il paraît avantageux de procéder à une coupure totale dans chacun des deux cas, en commencant par le bras sud.

Le premier projet envisagé consiste à couper le bras sud vers le milieu de l'lle au Héron en réalisant d'abord des digues à l'avancement dans les eaux peu profondes situées de part et d'autre du sillon central. Le débit sera alors concentré dans une zone rétrécie et profonde, d'une. largeur de 150 à $200 \mathrm{~m}$.

La coupure proprement dite serait ensuite réalisće à l'aide de gros tétraèdres (le chiffre de 20 tonnes a été envisagé) mis en place par couches horizontales successives à l'aide d'un blon-

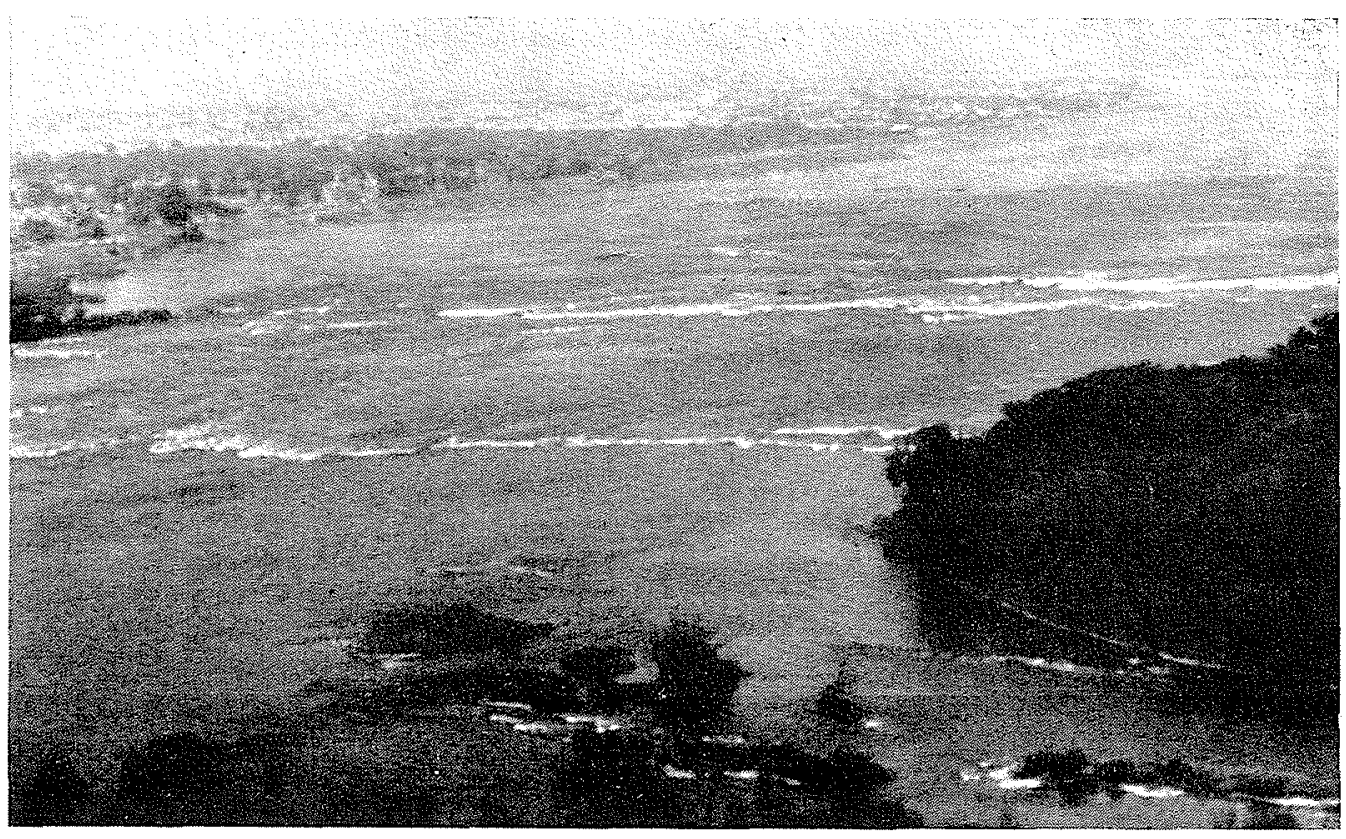

Fig. 14 .

L'Ile au Héron, bras nord du Saint-laarent et la Ville de Montréal. din. Chaque couche de tétraèdres serait complétée, avant mise en place de la suivante, par une couche d'enrochements de 1 tonne de facon à réduire le plus possible la consommation de tétraedres.

Une fois la coupure effectuée, l'étanchement est obtenu par aéversement, à l'amont des tétraćdres, d'abord de tout venant puis de matériaux iins.

La coupure du bras nord serail effectuée de manière similaire en réutilisant les tétraèdres récupérés dans le bras sud.

Ces méthodes font actuellement l'objet d'essais sur modèles au Laboratoire Hydraulique de La Salle, dont les liens techniques avec la SOGREAH sont connus. L'étude sur modele porte egalement sur une variante utilisant le seuil situé à hauteur de l'île du Diable; grâce à l'étalement du débit, une coupure enìièrement réalisée à l'avancement pourrait être envisagée.

\section{Problème des glaces.}

C'est le problème le plus original posé par cet aménagement.

Le Saint-Laurent gèle en hiver pendant trois mois environ. La surface des lacs est prise en premier et la couverture de glace remonte ensuite progressivement vers l'amont; elle crée des embâcles et des élévations de niveau dans les sections étroites du lit de la rivière. 
Fig. 15.

L'hélicoptère utilisé pour

les sondages.

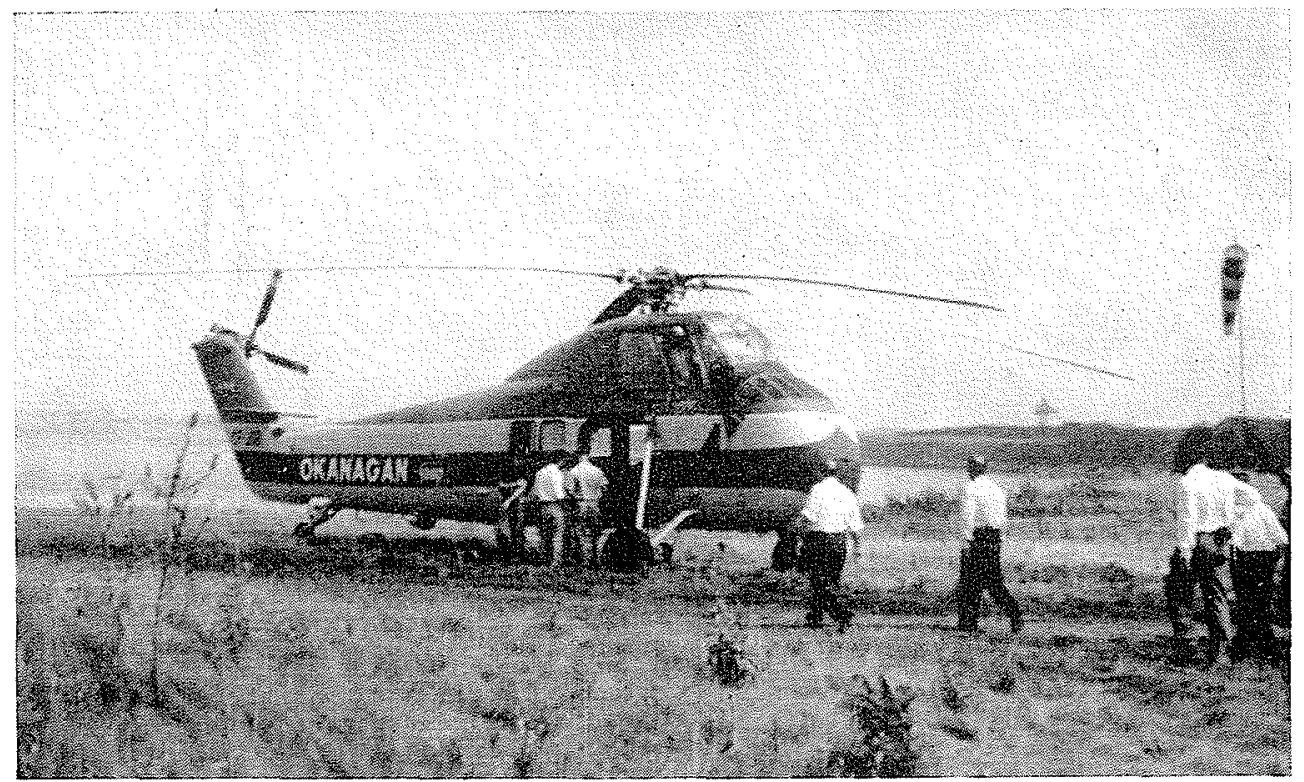

C'est ainsi que les niveaux d'hiver du SaintLaurent peuvent être sensiblement plus élevés que les niveaux en eaux libres de l'été.

Dans la portion du cours du Saint-Laurent qui intéresse Lachine, la couche de glace commence sur le lac Saint-Pierre, situé à une centaine de $\mathrm{km}$ en aval de Montréal, et remonte en un mois environ jusqu'au bassin de Laprairie et aux rapides.
Jusqu'à présent, la zone resserrée située entre le lac Saint-Louis et les rapides est toujours restée libre de glaces. Mais la construction de l'aménagement modifiera ces conditions en amorçant une couverture de glace à l'amont immédiat de l'usine.

Le problème le plus important est d'éviter l'élévation du niveau, à l'amont de la retenue, à une altitude telle qu'elle entraîne une inonda-
Fig. 16.

Emplacement de Lachine.

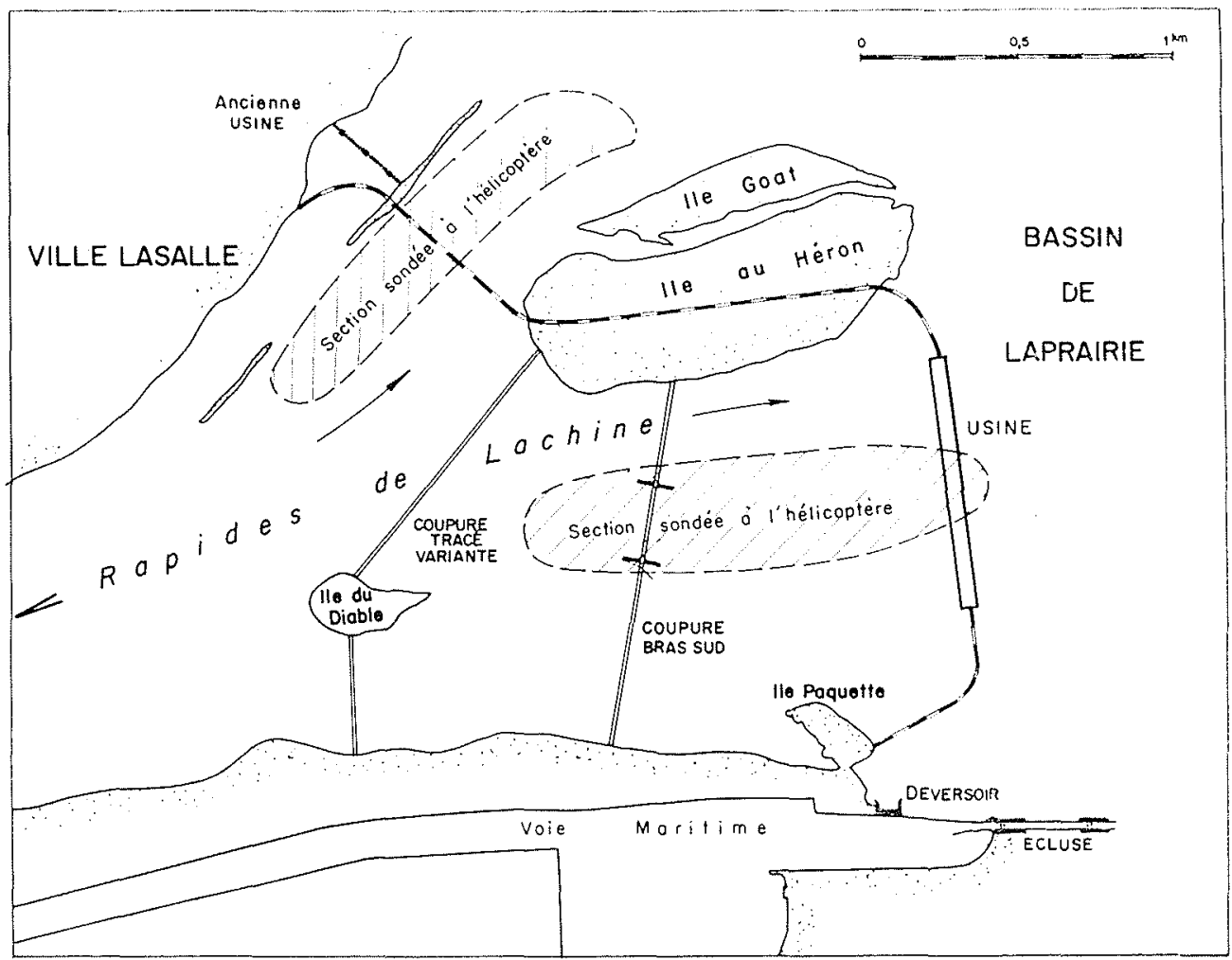






Fix. 17 .

Le Saint-Laurent en hiver a l'est de Montréal près de libe Sainte-Hélène.

tion des rives du lac Saint-Lonis et des agglomérations riveraines.

La régularisation du lac Ontario réduira les débits dans la période de formation des glaces à $7800 \mathrm{~m}^{3} / \mathrm{s}$, sur lesquels $1100 \mathrm{~m}^{3} / \mathrm{s}$ pourront être transités par la Voie Maritime; malgré cela, ies vitesses moyennes de l'eau sont un peu trop élevées dans les sections resserrées actuelles pour que l'on puisse espérer une remontée rapide de la couverture de glace, jusqu'au lac Saint-Louis.

Différentes idées ont été examinées:

-..- Division du cours en deux chenaux par une digue longitudinale afin de pouvoir jouer avec les vitesses moyennes dans chaque chenal et obtenir suceessivement la couverture de glace dans chacun;

-.- Installation d'estacades pour retenir les glaces dérivant du lac Saint-Louis et éviter ainsi la formation d'embâcles;

- Maintien d'un chenal à écoulement libre.
Pour réunir les éléments nécessaires à l'ctude l'une solution, des campagnes d'observation systématiques sont actuellement en cours, en pariiculier sur le canal de Beauharnois. Elles onl pour but d'observer les conditions de formation et de progression du couveri de glace, son maintien dans différentes conditions et le transport ct l'accumulation des glaces sous la couverture. Les incidences de la mise en place d'estacades ont également pu être observées avec précision.

Une autre série d'essais systématiques a été entreprise par MM. Cartier, Côté et Pielte dans un canal spécialement équipé près de l'ancienne usine de Lachine. Dans ce canal, les couverts on pu être formés et démolis à volonté en faisant varier les vitesses d'écoulement de l'eau.

Parallèlement à ces observations, une série d'études thériques a été entreprise au Laboratoire Hydraulique de La Salle; ces études portent notamment sur les conditions de propagation des éléments de glace sous les couverts.

Ces différentes études et observations se poursuivent achellement et feront probablement l'ob- 
jet de communications au Congrès qui doit se tenir en août 1959 à Montréal.

Pour obtenir une vitesse moyenne d'écoulement susceptible de permettre en toute circorstance la formation facile du couvert, il fauriait ćlargir considérablement les sections actuelles, sur les $8 \mathrm{~km}$ qui séparent l'extrémité du lac Saint-Louis de l'Ile au Héron.

La recherche de solutions permettant d'éviter ou de réduire cet élargissement présente done un intérèt économique considérable.

\section{DISCUSSION}

President : Mi. Duffavl

M. le president remercie M. Castrixal de sa conference et du film qui la suivie.

M. Desatrib, président de la S.H.F., fail les trois interventions ci-apres:

1. M. Detatre signale qu'a l'intérieur de lusine de Beauharnois, qui a maintenant plus de $1 / 2$ mille de longueur, la Direction a interdit la circulation a pied pour éviter les pertes de temps; elle se rait à seooter.

$2^{\circ}$ M. Delatroe indique qu'il a cu, à DonzèreMondragon, à considérer le problème des glaces. Non seulement on ne doit pas craindre l'embàcle (sauf' pour la circulation des bateaux, mais à Donzère cet arrêt de la navigation est rare et ne dure que quelques jours), mais on doit la provoquer en arrêtant l'usine une nuit, de manière a éviter la surcharge des grilles par des glaces flottant entre deux eaux, surcharge qui peut proroquer leur juplure.

Mais alors la retenue, moins profonde, fend at se prendre en masse of l'on doit craindre une surbevation de niveau due à l'effet de barrage des glaces; cette surclévation peut atteindre plusieurs metres of provoquer des débordements; c'est ce qui s'est proc'uit à Viviers au cours de l'hiver $1955-1956$.

La solution consiste à favoriser l'embâcle dans to canal et l'empêcner dans la retenue par des manouves adéquates du barrage de retenue.

M. Castelnay confinme l'importance du problème du charriage des glaces au Cantada.

Il précise que des surélévations, pouvant alteindre certaines annécs jusqu'à 5 ou 6 mètres par lapport au niveau normal d'été, se proc'uisent en hiver au port de Montréal, a cause des prises de graces qui ont lieu a l'aval.

La formation des embâcles dépend de la morphologio du lit : pour le Saint-Laurent, dans la région de Montréal, la couverture de glace commence à se former sur le lac Saint-Pierre, à une centaine de liblometres en aval de la ville. De là, la couverture remonte propressivement vers l'amont avec l'apport de glaces par le fleuve.

Dans les zonery où le lit est étriqué et les ritesses rapides, les glaces dérivantes, au lien de se coller a l'amont de la couverture, sont entrainées sous celle-ci et constituent parfois des barrages suspendus obstruant une grande partie de la section de passage de l'eau. L'obstacle ainsi crée produit à l'amont une surélévation du niveau el une réduction de la vitesse; lorsque celle-ci cst tombere a environ $0,60 \mathrm{~m} / \mathrm{s}$, les glaces d'apport ne sont plus entrainées sous le couvert et celui-ci reprend sa progression vers l'amont.

$3^{\circ}$ M. Dnatras demande pourguoi sur le SaintLaurent, on trouve indifféremment des usines "outdoor" ou "indoor", avec une nette prépondérance du dernier type, traditionnel, dans le Bassin du Columbia.

M. Castelnal pense que ladoption du type outdom (Bapnhart, usines récentes de lat région du lac Saint-Jean) répond a une idée d'économie; toutefois certains maitres d'xurres sont opposés it eetfe solution qui peut soulever des objections de la part des exploitants. Il n'y a done pas de doctrine établie of la solution retenue parait dépendre essentidlement du point de vue persomel du projeteur.

M. Lavgiors ajoute qu'il a eu l'occasion de poser la question par écrit et d'une manière précise en demandant si l'étude de la solution normale avait été faite et quel aurait été l'écart d'investissement entre les deux solutions. La réponse sera, bien entendu, communiquéc à la S.H.F.; on peut cependant être tenté de dire, at priori, que l'intérêt de l'outdoor par rapport au classique semble être essentiellement une question de nombre de groupes.

M. Chamayoy pense que les deux systèmes sont équivalents :

- D'abord comme investissement, si l'on traite les usines indoor aussi simplement que outdoor et sans souci architectural;

- Egalement comme entretien, si les groupes sont bien construits et ne demandent qu'une mise au point et un entretien raisonnables, ce vers quoi il faut tendre en définitive. 